\title{
Fluorescence Properties of Si-linked Oligothiophenes
}

\author{
Mamoru Fujitsuka, ${ }^{\dagger}$ Dae Won Cho, ${ }^{\dot{\dagger}, \#}$ Joji Ohshita, ${ }^{+}$Atsutaka Kunai, ${ }^{+}$and Tetsuro Majima ${ }^{\dagger *}$
}

\section{Synthetic methods and spectroscopic data of Si-linked oligothiophenes}

General Methods. All reactions were carried out in dry nitrogen. THF, ether, and toluene used as the reaction solvents were dried over sodium and distilled just before use. NMR spectra were recorded on a JEOL Model JNM-LA 400 spectrometer. FAB-MS spectra were obtained on a JEOL model SX102A spectrometer. 5 $\mathrm{T}_{2}$ was prepared as reported in the literature. ${ }^{1}$

Preparation of $3 \mathbf{T}_{3}$. To a solution of 5"-ethyl-5-lithio-2,2':5',2"-terthiophene ${ }^{2}$ prepared from $0.30 \mathrm{~g}(0.85 \mathrm{mmol})$ of 5-bromo-5"'-ethyl-2,2':5',2"-terthiophene and $0.59 \mathrm{~mL}(0.90 \mathrm{mmol})$ of a $1.52 \mathrm{M}$ of $n$-butyllithium/hexane solution in $3 \mathrm{~mL}$ of ether was added a solution of $0.80 \mathrm{~g}(0.25$ $\mathrm{mmol}$ ) of tris(chloromethylsilyl)methylsilane in $10 \mathrm{~mL}$ of $\mathrm{THF}$ at $-80^{\circ} \mathrm{C}$. The resulting mixture was allowed to warm to room temperature and was heated to reflux for $24 \mathrm{~h}$. After hydrolysis of the mixture, the organic layer was separated and the aqueous layer was extracted with ether. The organic layer and the extracts were combined and dried over anhydrous magnesium sulfate. After evaporation of the solvent, the residue was subjected to preparative GPC eluting with chloroform to give $0.15 \mathrm{~g}(0.14 \mathrm{mmol}, 56 \%$ yield $)$ of $3 \mathrm{~T}_{3}$ as the yellow solids: $\mathrm{mp} 126-127^{\circ} \mathrm{C}$; FAB-MS m/z $1042\left(\mathrm{M}^{+}\right) ;{ }^{1} \mathrm{H}$ NMR $\left(\delta\right.$ in $\left.\mathrm{CDCl}_{3}\right) 0.35\left(\mathrm{~s}, 3 \mathrm{H}, \mathrm{CH}_{3} \mathrm{Si}\right), 0.39\left(\mathrm{~s}, 18 \mathrm{H}, \mathrm{CH}_{3} \mathrm{Si}\right), 1.33(\mathrm{t}, 9 \mathrm{H}, J=7.6 \mathrm{~Hz}$, $\left.\mathrm{CH}_{3} \mathrm{CH}_{2}\right), 2.83\left(\mathrm{q} 6 \mathrm{H}, J=7.6 \mathrm{~Hz}, \mathrm{CH}_{2} \mathrm{CH}_{3}\right), 6.67$ (d, 3H, $J=3.5 \mathrm{~Hz}$, ring H), 6.92 (d, 3H, $J=3.4$ $\mathrm{Hz}$, ring $\mathrm{H}), 6.93$ (d, 3H, $J=3.5 \mathrm{~Hz}$, ring $\mathrm{H}), 6.94$ (d, 3H, $J=3.4 \mathrm{~Hz}$, ring H), 6.97 (d, 3H, $J=3.4$ $\mathrm{Hz}$, ring $\mathrm{H}), 7.11(\mathrm{~d}, 3 \mathrm{H}, J=3.4 \mathrm{~Hz}$, ring $\mathrm{H}) ;{ }^{13} \mathrm{C} \mathrm{NMR}\left(\delta\right.$ in $\left.\mathrm{CDCl}_{3}\right)-11.86\left(\mathrm{CH}_{3} \mathrm{Si}\right),-0.45\left(\mathrm{CH}_{3} \mathrm{Si}\right)$, $15.82\left(\mathrm{CH}_{3} \mathrm{CH}_{2}\right), 23.51\left(\mathrm{CH}_{2} \mathrm{CH}_{3}\right), 123.26,123.55,124.10,124.23,124.69,134.51,134.94,135.50$, 136.63, 138.78, 142.53, 146.86 (ring carbons). Anal. Calcd for $\mathrm{C}_{49} \mathrm{H}_{54} \mathrm{~S}_{9} \mathrm{Si}_{4}:$ C, 56.38; H, 5.21. Found: C, 56.12; H, 5.24.

Preparation of $\mathbf{5 T}_{3}$. A mixture of $0.10 \mathrm{~g} \quad(0.11 \mathrm{mmol})$ of $\operatorname{tris}\left[\left(5^{\prime}\right.\right.$-bromo-2,2'-bithienyl)dimethylsilyl]methylsilane, $\quad \begin{array}{lllll}0.25 & \mathrm{~g} & (0.44 & \mathrm{mmol}) & \text { of }\end{array}$ 5"-ethyl-5-(tributylstannyl)-2,2':5',2"-terthiophene, ${ }^{3} 3.8 \mathrm{mg}(0.0033 \mathrm{mmol})$ of $\mathrm{Pd}\left(\mathrm{PPh}_{3}\right)_{4}, 0.80 \mathrm{mg}$ $(0.0042 \mathrm{mmol})$ of $\mathrm{CuI}$, and $5 \mathrm{~mL}$ of toluene was heated at $110^{\circ} \mathrm{C}$ for $72 \mathrm{~h}$. The resulting orange solids were collected by filtration and were washed with ether $(0.11 \mathrm{~g}, 0.069 \mathrm{mmol}, 63 \%$ yield $)$ : $\mathrm{mp}$ 236 - $238^{\circ} \mathrm{C}$. Anal. Calcd for $\mathrm{C}_{73} \mathrm{H}_{66} \mathrm{~S}_{15} \mathrm{Si}_{4}: \mathrm{C}, 57.06 ; \mathrm{H}, 4.33$. Found: C, 57.09; H, 4.30. TOF-MS m/z $497\left(\mathrm{Et}\left(\mathrm{C}_{4} \mathrm{H}_{2} \mathrm{~S}\right)_{5} \mathrm{SiMe}_{2}{ }^{+}\right)$, IR (KBr) 1497 (Si-thiophene), 1250 (Si-Me) cm ${ }^{-1}$. NMR data could not be obtained, due to low solubility of the compound. 
Preparation of $3 \mathbf{T}_{2}$. To a solution of 5"-ethyl-5-lithio-2,2':5',2"-terthiophene ${ }^{2}$ prepared from $0.25 \mathrm{~g}(0.70 \mathrm{mmol})$ of 5-bromo-5"-ethyl-2,2':5',2"-terthiophene and $0.50 \mathrm{~mL}(0.70 \mathrm{mmol})$ of a $1.60 \mathrm{M}$ of $n$-butyllithium/hexane solution in $3 \mathrm{~mL}$ of ether was added $0.17 \mathrm{~g}(0.35 \mathrm{mmol})$ of 1,3-bis(trifluoromethanesulfonyl)hexamethyltrisilane at $-80^{\circ} \mathrm{C}$. The resulting mixture was allowed to warm to room temperature and was stirred for $24 \mathrm{~h}$. After hydrolysis of the mixture, the organic layer was separated and the aqueous layer was extracted with ether. The organic layer and the extracts were combined and dried over anhydrous magnesium sulfate. After evaporation of the solvent, the residue was recrystallized from hexane to give $0.11 \mathrm{~g}(0.16 \mathrm{mmol}, 46 \%$ yield $)$ of $3 \mathrm{~T}_{2}$ as the yellow solids: mp $110-111^{\circ} \mathrm{C}$; FAB-MS m/z $724\left(\mathrm{M}^{+}\right)$; ${ }^{1} \mathrm{H}$ NMR $\left(\delta\right.$ in $\left.\mathrm{CDCl}_{3}\right) 0.17(\mathrm{~s}, 6 \mathrm{H}$, $\mathrm{CH}_{3} \mathrm{Si}$ ), 0.36 (s, $\left.12 \mathrm{H}, \mathrm{CH}_{3} \mathrm{Si}\right), 1.31$ (t, $\left.6 \mathrm{H}, J=7.6 \mathrm{~Hz}, \mathrm{CH}_{3} \mathrm{CH}_{2}\right) 2.82$ (q, $4 \mathrm{H}, J=7.6 \mathrm{~Hz}, \mathrm{CH}_{2} \mathrm{CH}_{3}$ ), 6.67 (d, 2H, $J=3.6 \mathrm{~Hz}$, ring H), 6.94-6.95 (m, 4H, ring H), 6.98(d, 2H, $J=3.4 \mathrm{~Hz}$, ring H), 7.00 (d, $2 \mathrm{H}, J=3.6 \mathrm{~Hz}$, ring $\mathrm{H}), 7.15(\mathrm{~d}, 2 \mathrm{H}, J=3.4 \mathrm{~Hz}$, ring $\mathrm{H}) ;{ }^{13} \mathrm{C} \mathrm{NMR}\left(\delta\right.$ in $\left.\mathrm{CDCl}_{3}\right)-6.71\left(\mathrm{CH}_{3} \mathrm{Si}\right)$, -2.03 $\left(\mathrm{CH}_{3} \mathrm{Si}\right), 15.84\left(\mathrm{CH}_{3} \mathrm{CH}_{2}\right), 23.51\left(\mathrm{CH}_{2} \mathrm{CH}_{3}\right), 123.28,123.56,124.11,124.20,124.79,134.50$, 134.88, 135.55, 136.63, 138.47, 142.36, 146.95 (ring carbons). Anal. Calcd for $\mathrm{C}_{34} \mathrm{H}_{40} \mathrm{~S}_{6} \mathrm{Si}_{3}: \mathrm{C}$, 56.30; H, 5.56. Found: C, 56.33; H, 5.38.

Preparation of $3 \mathbf{T}_{1}$. To a solution of 5"-ethyl-5-lithio-2,2':5',2"-terthiophene ${ }^{2}$ prepared from $0.130 \mathrm{~g}(0.28 \mathrm{mmol})$ of 5-bromo-5"-ethyl-2,2':5',2"-terthiophene and $0.18 \mathrm{~mL}(0.29 \mathrm{mmol})$ of a $1.59 \mathrm{M}$ of $n$-butyllithium/hexane solution in $3 \mathrm{~mL}$ of ether was added $0.034 \mathrm{~g}(0.31 \mathrm{mmol})$ of trimethylchlorosilane at $-80^{\circ} \mathrm{C}$. The resulting mixture was allowed to warm to room temperature and was stirred for $3 \mathrm{~h}$. After hydrolysis of the mixture, the organic layer was separated and the aqueous layer was extracted with ether. The organic layer and the extracts were combined and dried over anhydrous magnesium sulfate. After evaporation of the solvent, the residue was subjected to preparative GPC eluting with chloroform to give $0.064 \mathrm{~g}(0.18 \mathrm{mmol}, 64 \%$ yield $)$ of $3 \mathrm{~T}_{1}$ as the yellow solids: $\mathrm{mp} 68-70^{\circ} \mathrm{C}$; $\mathrm{MS} \mathrm{m} / \mathrm{z} 348\left(\mathrm{M}^{+}\right) ;{ }^{1} \mathrm{H} \mathrm{NMR}\left(\delta\right.$ in $\left.\mathrm{CDCl}_{3}\right) 0.32(\mathrm{~s}, 9 \mathrm{H}$, $\mathrm{Me}_{3} \mathrm{Si}$ ), 1.31 (t, 6H, $\left.J=7.6 \mathrm{~Hz}, \mathrm{CH}_{3} \mathrm{CH}_{2}\right), 2.78$ (q, 4H, $\left.J=7.6 \mathrm{~Hz}, \mathrm{CH}_{2} \mathrm{CH}_{3}\right), 6.69$ (d, $1 \mathrm{H}, J=3.7$ $\mathrm{Hz}$, ring $\mathrm{H}), 6.97$ (d, $1 \mathrm{H}, J=3.5 \mathrm{~Hz}$, ring $\mathrm{H}), 6.98(\mathrm{~d}, 1 \mathrm{H}, J=3.7 \mathrm{~Hz}$, ring $\mathrm{H}), 7.05$ (d, $1 \mathrm{H}, J=3.7$ $\mathrm{Hz}$, ring $\mathrm{H}), 7.12(\mathrm{~d}, 1 \mathrm{H}, J=3.7 \mathrm{~Hz}$, ring $\mathrm{H}), 7.19(\mathrm{~d}, 1 \mathrm{H}, J=3.5 \mathrm{~Hz}$, ring $\mathrm{H}) ;{ }^{13} \mathrm{C} \mathrm{NMR}(\delta$ in $\left.\mathrm{CDCl}_{3}\right)-0.13\left(\mathrm{Me}_{3} \mathrm{Si}\right), 15.84\left(\mathrm{CH}_{3} \mathrm{CH}_{2}\right), 23.52\left(\mathrm{CH}_{2} \mathrm{CH}_{3}\right), 123.32,123.56,124.13,124.30,124.73$, 134.44, 134.75, 135.52, 136.78, 138.72, 142.21, 147.04 (ring carbons). Anal. Calcd for $\mathrm{C}_{17} \mathrm{H}_{20} \mathrm{~S}_{3} \mathrm{Si}: \mathrm{C}, 58.57$; H, 5.78. Found: C, 58.62; H, 5.59. 
Energy-minimized structures of $5 T_{3}$ and $3 T_{2}$

(a)

(b)
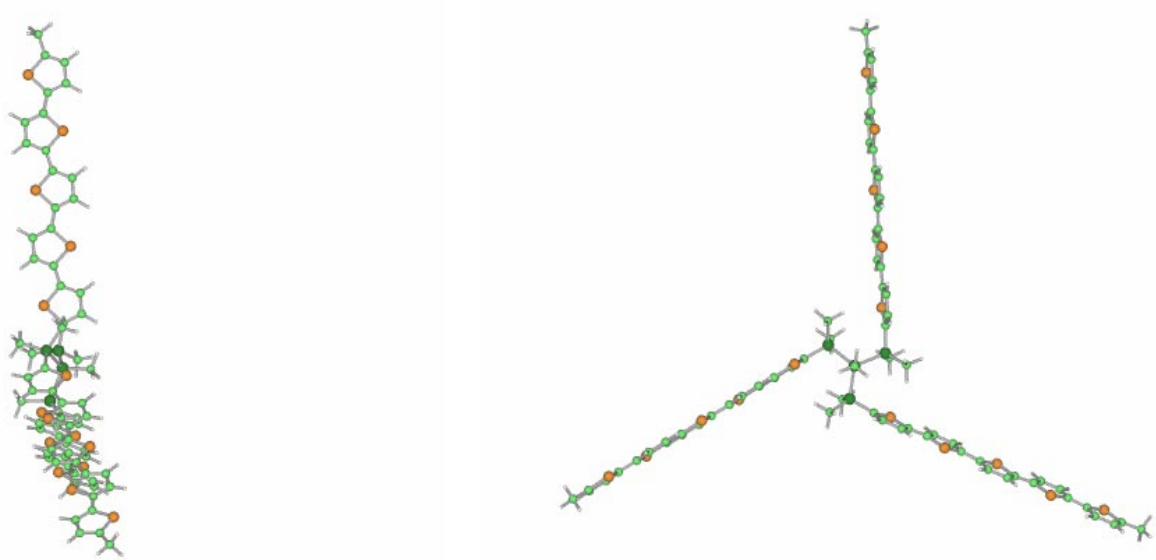

(c)

(d)
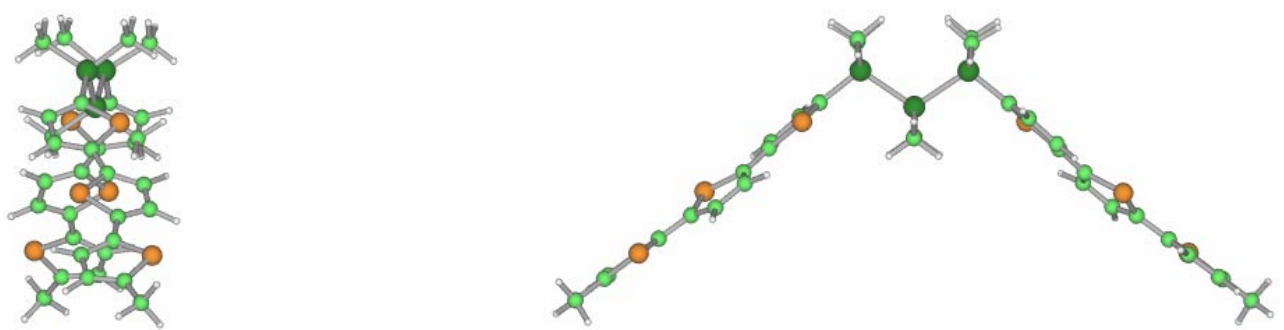

Figure S1. Optimized structures of $5 \mathrm{~T}_{3}$ and $3 \mathrm{~T}_{2}$ (side view $(\mathrm{a}, \mathrm{c}$ ) and top view (b, d)) at B3LYP/3-21G* level. In the theoretical calculation, ethyl groups at the $\alpha$-position of oligothiophenes were reduced to methyl groups for the simplicity of the calculation. 
Energy-minimized structures of Si-linked oligothiophenes at B3LYP/3-21G* level.

$3 \mathrm{~T}_{1}$ (Sum of electronic and thermal Enthalpies $=-2093.946369$ Hartree $)$

\begin{tabular}{|c|c|c|c|}
\hline & $\mathrm{X}$ & $\mathrm{Y}$ & $\mathrm{Z}$ \\
\hline $\mathrm{Si}$ & -5.63154 & -0.10261 & -0.03257 \\
\hline $\mathrm{C}$ & -6.10802 & -0.97282 & 1.575298 \\
\hline $\mathrm{C}$ & -5.98466 & -1.24843 & -1.4929 \\
\hline $\mathrm{C}$ & -6.58978 & 1.514454 & -0.21646 \\
\hline $\mathrm{C}$ & 8.011269 & -0.31753 & -0.21709 \\
\hline $\mathrm{C}$ & 6.568854 & 0.09583 & -0.09919 \\
\hline $\mathrm{C}$ & 6.062354 & 1.360562 & 0.061672 \\
\hline $\mathrm{C}$ & 4.638103 & 1.399982 & 0.145848 \\
\hline $\mathrm{C}$ & 4.048978 & 0.156745 & 0.046587 \\
\hline $\mathrm{S}$ & 5.279249 & -1.08148 & -0.16274 \\
\hline $\mathrm{C}$ & 0.120934 & -0.1301 & 0.100415 \\
\hline $\mathrm{C}$ & 0.648306 & -1.39587 & 0.271379 \\
\hline $\mathrm{C}$ & 2.066783 & -1.42668 & 0.268638 \\
\hline $\mathrm{C}$ & 2.646944 & -0.18473 & 0.092559 \\
\hline $\mathrm{S}$ & 1.409965 & 1.045991 & -0.09143 \\
\hline $\mathrm{C}$ & -1.26543 & 0.270167 & 0.062094 \\
\hline $\mathrm{C}$ & -1.7905 & 1.548188 & 0.02403 \\
\hline $\mathrm{C}$ & -3.21378 & 1.569966 & -0.00999 \\
\hline $\mathrm{C}$ & -3.80576 & 0.320128 & -0.00139 \\
\hline $\mathrm{S}$ & -2.55994 & -0.91216 & 0.049542 \\
\hline $\mathrm{H}$ & -7.17485 & -1.23554 & 1.578377 \\
\hline $\mathrm{H}$ & -5.91436 & -0.33017 & 2.443649 \\
\hline $\mathrm{H}$ & -5.53494 & -1.89951 & 1.712182 \\
\hline $\mathrm{H}$ & -5.39954 & -2.17545 & -1.42605 \\
\hline $\mathrm{H}$ & -5.73201 & -0.76326 & -2.4444 \\
\hline $\mathrm{H}$ & -7.04708 & -1.52647 & -1.52527 \\
\hline $\mathrm{H}$ & -6.40038 & 2.192722 & 0.625796 \\
\hline $\mathrm{H}$ & -7.6701 & 1.319463 & -0.24885 \\
\hline $\mathrm{H}$ & -6.31725 & 2.036364 & -1.14286 \\
\hline $\mathrm{H}$ & 8.302709 & -0.9972 & 0.592894 \\
\hline $\mathrm{H}$ & 8.649347 & 0.569865 & -0.16346 \\
\hline $\mathrm{H}$ & 8.208954 & -0.82287 & -1.17023 \\
\hline $\mathrm{H}$ & 6.689673 & 2.239258 & 0.124636 \\
\hline $\mathrm{H}$ & 4.071365 & 2.309528 & 0.2922 \\
\hline $\mathrm{H}$ & 0.032262 & -2.27344 & 0.411969 \\
\hline $\mathrm{H}$ & 2.645098 & -2.3291 & 0.411983 \\
\hline $\mathrm{H}$ & -1.17652 & 2.438969 & 0.029353 \\
\hline $\mathrm{H}$ & -3.78668 & 2.486622 & -0.04093 \\
\hline & & & \\
\hline
\end{tabular}

$3 \mathrm{~T}_{2}$ (Sum of electronic and thermal Enthalpies $=-4475.956954$ Hartree $)$

\begin{tabular}{|l|c|c|c|}
\hline & $\mathrm{X}$ & $\mathrm{Y}$ & $\mathrm{Z}$ \\
\hline $\mathrm{C}$ & 12.68533 & -4.76024 & -0.7122 \\
\hline $\mathrm{C}$ & 11.49932 & -3.99141 & -0.19512 \\
\hline $\mathrm{C}$ & 11.054 & -3.89359 & 1.09896 \\
\hline $\mathrm{C}$ & 9.8866 & -3.08543 & 1.24257 \\
\hline $\mathrm{C}$ & 9.43567 & -2.55804 & 0.05034 \\
\hline $\mathrm{S}$ & 10.47959 & -3.0651 & -1.27015 \\
\hline $\mathrm{C}$ & 6.30426 & -0.16838 & 0.05202 \\
\hline $\mathrm{C}$ & 6.55482 & -0.55132 & -1.2522 \\
\hline $\mathrm{C}$ & 7.67074 & -1.41671 & -1.3869 \\
\hline $\mathrm{C}$ & 8.29207 & -1.70943 & -0.18746 \\
\hline $\mathrm{S}$ & 7.49087 & -0.87806 & 1.13385 \\
\hline $\mathrm{C}$ & 5.25305 & 0.68528 & 0.55142 \\
\hline $\mathrm{C}$ & 4.85434 & 0.88736 & 1.86004 \\
\hline $\mathrm{C}$ & 3.77405 & 1.80589 & 1.98354 \\
\hline $\mathrm{C}$ & 3.32635 & 2.32579 & 0.78118 \\
\hline $\mathrm{S}$ & 4.27716 & 1.65896 & -0.53195 \\
\hline $\mathrm{Si}$ & 1.88663 & 3.49085 & 0.47785 \\
\hline $\mathrm{Si}$ & -1.88661 & 3.4908 & -0.47784 \\
\hline $\mathrm{C}$ & 1.63458 & 4.50488 & 2.06064 \\
\hline $\mathrm{C}$ & -2.33483 & 4.63918 & 0.96368 \\
\hline $\mathrm{C}$ & 2.33485 & 4.63931 & -0.96361 \\
\hline $\mathrm{C}$ & -1.63463 & 4.50492 & -2.06059 \\
\hline $\mathrm{C}$ & -12.6854 & -4.76017 & 0.71219 \\
\hline $\mathrm{C}$ & -11.4994 & -3.99138 & 0.19511 \\
\hline $\mathrm{C}$ & -11.054 & -3.89361 & -1.09895 \\
\hline
\end{tabular}




\begin{tabular}{|c|c|c|c|}
\hline $\mathrm{C}$ & -9.88658 & -3.08547 & -1.24255 \\
\hline $\mathrm{C}$ & -9.43569 & -2.55804 & -0.05033 \\
\hline $\mathrm{S}$ & -10.4796 & -3.06506 & 1.27015 \\
\hline $\mathrm{C}$ & -6.30426 & -0.1684 & -0.05201 \\
\hline $\mathrm{C}$ & -6.5548 & -0.55136 & 1.25221 \\
\hline $\mathrm{C}$ & -7.67072 & -1.41675 & 1.38691 \\
\hline $\mathrm{C}$ & -8.29209 & -1.70942 & 0.18747 \\
\hline $\mathrm{S}$ & -7.49099 & -0.87793 & -1.13382 \\
\hline $\mathrm{C}$ & -5.25303 & 0.68524 & -0.55141 \\
\hline $\mathrm{C}$ & -4.85428 & 0.88726 & -1.86003 \\
\hline $\mathrm{C}$ & -3.77398 & 1.80578 & -1.98354 \\
\hline $\mathrm{C}$ & -3.32631 & 2.32572 & -0.78118 \\
\hline $\mathrm{S}$ & -4.27717 & 1.65895 & 0.53195 \\
\hline $\mathrm{Si}$ & 0.00004 & 2.18774 & -0.00005 \\
\hline $\mathrm{C}$ & 0.36184 & 1.08544 & -1.51191 \\
\hline $\mathrm{C}$ & -0.36171 & 1.0853 & 1.51172 \\
\hline $\mathrm{H}$ & 13.40862 & -4.10099 & -1.20748 \\
\hline $\mathrm{H}$ & 12.38299 & -5.53106 & -1.43161 \\
\hline $\mathrm{H}$ & 13.19126 & -5.25389 & 0.1232 \\
\hline $\mathrm{H}$ & 11.54257 & -4.39136 & 1.92561 \\
\hline $\mathrm{H}$ & 9.38723 & -2.91525 & 2.18684 \\
\hline $\mathrm{H}$ & 5.93905 & -0.23619 & -2.08349 \\
\hline $\mathrm{H}$ & 7.99461 & -1.83271 & -2.33111 \\
\hline $\mathrm{H}$ & 5.31419 & 0.38378 & 2.69999 \\
\hline $\mathrm{H}$ & 3.33738 & 2.0769 & 2.93532 \\
\hline $\mathrm{H}$ & 0.81557 & 5.225 & 1.93378 \\
\hline $\mathrm{H}$ & 2.54433 & 5.06413 & 2.31503 \\
\hline $\mathrm{H}$ & 1.37928 & 3.86155 & 2.91265 \\
\hline $\mathrm{H}$ & -2.53792 & 4.0737 & 1.88287 \\
\hline $\mathrm{H}$ & -3.22957 & 5.22956 & 0.72635 \\
\hline $\mathrm{H}$ & -1.51381 & 5.33584 & 1.17894 \\
\hline $\mathrm{H}$ & 1.5138 & 5.33594 & -1.17887 \\
\hline $\mathrm{H}$ & 2.53798 & 4.07387 & -1.88281 \\
\hline $\mathrm{H}$ & 3.22955 & 5.22971 & -0.72623 \\
\hline $\mathrm{H}$ & -1.37936 & 3.86162 & -2.91265 \\
\hline $\mathrm{H}$ & -0.81561 & 5.22504 & -1.93372 \\
\hline $\mathrm{H}$ & -2.54439 & 5.06417 & -2.31491 \\
\hline $\mathrm{H}$ & -12.3831 & -5.531 & 1.4316 \\
\hline $\mathrm{H}$ & -13.1913 & -5.2538 & -0.12321 \\
\hline $\mathrm{H}$ & -13.4087 & -4.1009 & 1.20745 \\
\hline $\mathrm{H}$ & -11.5426 & -4.39139 & -1.9256 \\
\hline $\mathrm{H}$ & -9.38718 & -2.91533 & -2.18681 \\
\hline $\mathrm{H}$ & -5.93898 & -0.23629 & 2.08348 \\
\hline $\mathrm{H}$ & -7.99454 & -1.83282 & 2.33111 \\
\hline $\mathrm{H}$ & -5.3141 & 0.38363 & -2.69998 \\
\hline $\mathrm{H}$ & -3.33728 & 2.07676 & -2.93531 \\
\hline $\mathrm{H}$ & -0.50045 & 0.4424 & -1.73158 \\
\hline $\mathrm{H}$ & 1.23287 & 0.44465 & -1.32364 \\
\hline $\mathrm{H}$ & 0.57246 & 1.6897 & -2.40401 \\
\hline $\mathrm{H}$ & -1.23271 & 0.44448 & 1.32341 \\
\hline $\mathrm{H}$ & -0.57235 & 1.68948 & 2.40388 \\
\hline $\mathrm{H}$ & 0.50062 & 0.44229 & 1.73134 \\
\hline
\end{tabular}

$3 \mathrm{~T}_{3}$ (Sum of electronic and thermal Enthalpies $=-6490.577179$ Hartree $)$

\begin{tabular}{|l|c|c|c|}
\hline & $\mathrm{X}$ & $\mathrm{Y}$ & $\mathrm{Z}$ \\
\hline $\mathrm{C}$ & 1.64469 & 12.46992 & -1.24569 \\
\hline $\mathrm{C}$ & 1.26424 & 11.09975 & -1.36175 \\
\hline $\mathrm{C}$ & 0.87155 & 10.53581 & -0.16528 \\
\hline $\mathrm{S}$ & 0.97543 & 11.72497 & 1.12632 \\
\hline $\mathrm{C}$ & 1.54654 & 12.96134 & 0.03146 \\
\hline $\mathrm{C}$ & -0.47128 & 4.92328 & -1.84247 \\
\hline $\mathrm{C}$ & -0.86176 & 3.55749 & -1.93728 \\
\hline $\mathrm{C}$ & -1.24874 & 2.98836 & -0.73607 \\
\hline $\mathrm{S}$ & -1.13485 & 4.18707 & 0.5376 \\
\hline $\mathrm{C}$ & -0.2517 & 6.75974 & -0.09148 \\
\hline $\mathrm{C}$ & -0.34387 & 7.26491 & 1.19198 \\
\hline $\mathrm{C}$ & 0.03667 & 8.62675 & 1.29698 \\
\hline $\mathrm{C}$ & 0.42678 & 9.18813 & 0.09543 \\
\hline $\mathrm{S}$ & 0.32201 & 8.00091 & -1.19355 \\
\hline $\mathrm{C}$ & -0.55629 & 5.42864 & -0.55702 \\
\hline $\mathrm{Si}$ & -1.76815 & 1.21399 & -0.39268 \\
\hline $\mathrm{C}$ & 1.86212 & 14.34977 & 0.51872 \\
\hline
\end{tabular}




\begin{tabular}{|c|c|c|c|}
\hline $\mathrm{C}$ & -3.02182 & 0.70203 & -1.71624 \\
\hline $\mathrm{C}$ & -2.56332 & 1.15697 & 1.32533 \\
\hline $\mathrm{C}$ & -11.5317 & -5.24988 & -1.42961 \\
\hline $\mathrm{C}$ & -10.1385 & -4.94406 & -1.40621 \\
\hline $\mathrm{C}$ & -9.69346 & -4.50417 & -0.17637 \\
\hline $\mathrm{S}$ & -11.0258 & -4.46834 & 0.97094 \\
\hline $\mathrm{C}$ & -12.1592 & -5.04764 & -0.22641 \\
\hline $\mathrm{C}$ & -3.8695 & -3.5349 & -1.27589 \\
\hline $\mathrm{C}$ & -2.48019 & -3.22756 & -1.23678 \\
\hline $\mathrm{C}$ & -2.02284 & -2.80134 & -0.00118 \\
\hline $\mathrm{S}$ & -3.35353 & -2.79286 & 1.13869 \\
\hline $\mathrm{C}$ & -5.89423 & -3.55963 & 0.26765 \\
\hline $\mathrm{C}$ & -6.5334 & -3.36352 & 1.47743 \\
\hline $\mathrm{C}$ & -7.91726 & -3.6717 & 1.44719 \\
\hline $\mathrm{C}$ & -8.3615 & -4.10894 & 0.21319 \\
\hline $\mathrm{S}$ & -7.03472 & -4.13864 & -0.93582 \\
\hline $\mathrm{C}$ & -4.50545 & -3.34809 & -0.06121 \\
\hline $\mathrm{Si}$ & -0.28841 & -2.24487 & 0.46693 \\
\hline $\mathrm{C}$ & -13.6092 & -5.26532 & 0.11202 \\
\hline $\mathrm{C}$ & 0.92785 & -3.54127 & -0.18427 \\
\hline $\mathrm{C}$ & -0.18454 & -2.13645 & 2.35516 \\
\hline $\mathrm{C}$ & 10.29 & -7.46987 & 1.06742 \\
\hline $\mathrm{C}$ & 9.27895 & -6.47477 & 1.21918 \\
\hline $\mathrm{C}$ & 8.86778 & -5.92295 & 0.02321 \\
\hline $\mathrm{S}$ & 9.75053 & -6.65015 & -1.31289 \\
\hline $\mathrm{C}$ & 10.65768 & -7.6855 & -0.23663 \\
\hline $\mathrm{C}$ & 4.72991 & -1.97854 & 1.86769 \\
\hline $\mathrm{C}$ & 3.72346 & -0.97953 & 2.0024 \\
\hline $\mathrm{C}$ & 3.30283 & -0.42699 & 0.80504 \\
\hline $\mathrm{S}$ & 4.1828 & -1.16814 & -0.51819 \\
\hline $\mathrm{C}$ & 6.08216 & -3.13677 & 0.04771 \\
\hline $\mathrm{C}$ & 6.45748 & -3.36236 & -1.2633 \\
\hline $\mathrm{C}$ & 7.46279 & -4.35234 & -1.40574 \\
\hline $\mathrm{C}$ & 7.87449 & -4.90162 & -0.20574 \\
\hline $\mathrm{S}$ & 6.99657 & -4.17467 & 1.1294 \\
\hline $\mathrm{C}$ & 5.0991 & -2.21069 & 0.55543 \\
\hline $\mathrm{Si}$ & 1.97518 & 0.86992 & 0.51106 \\
\hline $\mathrm{C}$ & 11.68323 & -8.65212 & -0.76437 \\
\hline $\mathrm{C}$ & 2.68147 & 2.21391 & -0.62041 \\
\hline $\mathrm{C}$ & 1.52275 & 1.59969 & 2.19879 \\
\hline $\mathrm{Si}$ & 0.13255 & -0.15762 & -0.51969 \\
\hline $\mathrm{C}$ & 0.5494 & -0.42892 & -2.37415 \\
\hline $\mathrm{H}$ & 1.97958 & 13.07064 & -2.08038 \\
\hline $\mathrm{H}$ & 1.28043 & 10.55128 & -2.29399 \\
\hline $\mathrm{H}$ & -0.14597 & 5.51452 & -2.68826 \\
\hline $\mathrm{H}$ & -0.8588 & 3.00787 & -2.86937 \\
\hline $\mathrm{H}$ & -0.67791 & 6.67265 & 2.03295 \\
\hline $\mathrm{H}$ & 0.0236 & 9.17992 & 2.22619 \\
\hline $\mathrm{H}$ & 2.21216 & 14.95905 & -0.3202 \\
\hline $\mathrm{H}$ & 2.64787 & 14.33953 & 1.28392 \\
\hline $\mathrm{H}$ & 0.9781 & 14.83664 & 0.9484 \\
\hline $\mathrm{H}$ & -3.3283 & -0.34199 & -1.57272 \\
\hline $\mathrm{H}$ & -2.59718 & 0.79354 & -2.72452 \\
\hline $\mathrm{H}$ & -3.91536 & 1.33793 & -1.66641 \\
\hline $\mathrm{H}$ & -2.93458 & 0.14511 & 1.53226 \\
\hline $\mathrm{H}$ & -3.41793 & 1.84475 & 1.38111 \\
\hline $\mathrm{H}$ & -1.85486 & 1.42775 & 2.11914 \\
\hline $\mathrm{H}$ & -12.051 & -5.60779 & -2.30828 \\
\hline $\mathrm{H}$ & -9.48933 & -5.04574 & -2.26558 \\
\hline $\mathrm{H}$ & -4.38257 & -3.88323 & -2.16252 \\
\hline $\mathrm{H}$ & -1.83243 & -3.32055 & -2.09871 \\
\hline $\mathrm{H}$ & -6.02052 & -3.00792 & 2.36064 \\
\hline $\mathrm{H}$ & -8.56857 & -3.57599 & 2.30525 \\
\hline $\mathrm{H}$ & -14.1388 & -5.629 & -0.77389 \\
\hline $\mathrm{H}$ & -14.0907 & -4.33582 & 0.43954 \\
\hline $\mathrm{H}$ & -13.7296 & -6.00793 & 0.9102 \\
\hline $\mathrm{H}$ & 1.96173 & -3.24741 & 0.03601 \\
\hline $\mathrm{H}$ & 0.83801 & -3.66092 & -1.2716 \\
\hline $\mathrm{H}$ & 0.73526 & -4.51714 & 0.28049 \\
\hline $\mathrm{H}$ & 0.84105 & -1.87874 & 2.64937 \\
\hline $\mathrm{H}$ & -0.43804 & -3.0982 & 2.82091 \\
\hline $\mathrm{H}$ & -0.85726 & -1.3707 & 2.763 \\
\hline $\mathrm{H}$ & 10.72951 & -8.00744 & 1.89662 \\
\hline $\mathrm{H}$ & 8.87066 & -6.17811 & 2.17598 \\
\hline
\end{tabular}




\begin{tabular}{|c|c|c|c|}
\hline $\mathrm{H}$ & 5.17034 & -2.50231 & 2.70576 \\
\hline $\mathrm{H}$ & 3.32643 & -0.66843 & 2.95925 \\
\hline $\mathrm{H}$ & 6.02209 & -2.82986 & -2.09784 \\
\hline $\mathrm{H}$ & 7.87267 & -4.65321 & -2.36024 \\
\hline $\mathrm{H}$ & 12.12637 & -9.20423 & 0.0701 \\
\hline $\mathrm{H}$ & 12.48974 & -8.13302 & -1.29656 \\
\hline $\mathrm{H}$ & 11.23545 & -9.37801 & -1.45404 \\
\hline $\mathrm{H}$ & 1.92581 & 2.98149 & -0.82948 \\
\hline $\mathrm{H}$ & 3.0115 & 1.79559 & -1.58049 \\
\hline $\mathrm{H}$ & 3.5472 & 2.69767 & -0.14894 \\
\hline $\mathrm{H}$ & 2.41206 & 2.01318 & 2.69329 \\
\hline $\mathrm{H}$ & 1.07509 & 0.85133 & 2.86507 \\
\hline $\mathrm{H}$ & 0.8011 & 2.41644 & 2.06909 \\
\hline $\mathrm{H}$ & 1.43735 & -1.0643 & -2.48398 \\
\hline $\mathrm{H}$ & 0.74998 & 0.52924 & -2.8702 \\
\hline $\mathrm{H}$ & -0.28734 & -0.91488 & -2.89217 \\
\hline
\end{tabular}

$5 \mathrm{~T}_{2}$ (Sum of electronic and thermal Enthalpies $=-6461.092047$ Hartree $)$

\begin{tabular}{|c|c|c|c|}
\hline & $\mathrm{X}$ & $\mathrm{Y}$ & $\mathrm{Z}$ \\
\hline $\mathrm{C}$ & -17.7916 & -0.62823 & -0.72139 \\
\hline $\mathrm{C}$ & -18.3756 & -1.76181 & -0.19334 \\
\hline $\mathrm{C}$ & -19.7836 & -1.82817 & -0.41039 \\
\hline $\mathrm{C}$ & -20.2845 & -0.75361 & -1.10123 \\
\hline $\mathrm{S}$ & -19.0095 & 0.37174 & -1.50197 \\
\hline $\mathrm{C}$ & -16.4055 & -0.23097 & -0.69315 \\
\hline $\mathrm{C}$ & -15.8235 & 0.90874 & -1.21774 \\
\hline $\mathrm{C}$ & -14.4242 & 0.98175 & -1.00763 \\
\hline $\mathrm{C}$ & -13.9087 & -0.10146 & -0.31833 \\
\hline $\mathrm{S}$ & -15.1902 & -1.23609 & 0.0778 \\
\hline $\mathrm{C}$ & -10.0487 & -0.22676 & 0.44225 \\
\hline $\mathrm{C}$ & -10.6313 & -1.3695 & 0.9626 \\
\hline $\mathrm{C}$ & -12.0295 & -1.44192 & 0.7519 \\
\hline $\mathrm{C}$ & -12.5454 & -0.35618 & 0.06536 \\
\hline $\mathrm{S}$ & -11.265 & 0.77981 & -0.32755 \\
\hline $\mathrm{C}$ & -8.66618 & 0.17086 & 0.47363 \\
\hline $\mathrm{C}$ & -8.08533 & 1.31691 & -0.04112 \\
\hline $\mathrm{C}$ & -6.68663 & 1.38913 & 0.1698 \\
\hline $\mathrm{C}$ & -6.16964 & 0.30054 & 0.84865 \\
\hline $\mathrm{S}$ & -7.448 & -0.83889 & 1.23556 \\
\hline $\mathrm{C}$ & -2.29358 & 0.18548 & 1.61805 \\
\hline $\mathrm{C}$ & -2.88033 & -0.96803 & 2.10971 \\
\hline $\mathrm{C}$ & -4.28457 & -1.05082 & 1.89508 \\
\hline $\mathrm{C}$ & -4.80238 & 0.04512 & 1.22873 \\
\hline $\mathrm{S}$ & -3.52221 & 1.19053 & 0.87263 \\
\hline $\mathrm{C}$ & -21.7106 & -0.492 & -1.50327 \\
\hline $\mathrm{Si}$ & -0.47879 & 0.67025 & 1.63873 \\
\hline $\mathrm{Si}$ & 0.32943 & 0.47672 & -0.5603 \\
\hline $\mathrm{C}$ & 0.37733 & -0.44781 & 2.91645 \\
\hline $\mathrm{C}$ & -0.33874 & 2.4909 & 2.17432 \\
\hline $\mathrm{C}$ & -0.86217 & 2.74725 & 3.61568 \\
\hline $\mathrm{C}$ & 1.85592 & -0.05017 & 3.19343 \\
\hline $\mathrm{C}$ & -0.45508 & 1.86806 & -1.59824 \\
\hline $\mathrm{C}$ & -0.06013 & 1.82083 & -3.10202 \\
\hline $\mathrm{C}$ & -0.11229 & -1.20133 & -1.33335 \\
\hline $\mathrm{C}$ & 0.26254 & -2.45157 & -0.48984 \\
\hline $\mathrm{C}$ & 17.83888 & -0.36489 & -0.28084 \\
\hline $\mathrm{C}$ & 18.48503 & 0.85204 & -0.35951 \\
\hline $\mathrm{C}$ & 19.90659 & 0.74258 & -0.32741 \\
\hline $\mathrm{C}$ & 20.35617 & -0.54976 & -0.22486 \\
\hline $\mathrm{S}$ & 19.01477 & -1.66768 & -0.16642 \\
\hline $\mathrm{C}$ & 16.42239 & -0.63587 & -0.28494 \\
\hline $\mathrm{C}$ & 15.77699 & -1.85691 & -0.21104 \\
\hline $\mathrm{C}$ & 14.36408 & -1.75677 & -0.24091 \\
\hline $\mathrm{C}$ & 13.90124 & -0.45649 & -0.33867 \\
\hline $\mathrm{S}$ & 15.25089 & 0.66695 & -0.39423 \\
\hline $\mathrm{C}$ & 10.0207 & 0.19326 & -0.4471 \\
\hline $\mathrm{C}$ & 10.66806 & 1.41425 & -0.5244 \\
\hline $\mathrm{C}$ & 12.07969 & 1.31281 & -0.49395 \\
\hline $\mathrm{C}$ & 12.54184 & 0.01174 & -0.39343 \\
\hline $\mathrm{S}$ & 11.19221 & -1.11052 & -0.33231 \\
\hline $\mathrm{C}$ & 8.60721 & -0.07564 & -0.45158 \\
\hline $\mathrm{C}$ & 7.96029 & -1.29821 & -0.39994 \\
\hline
\end{tabular}




\begin{tabular}{|c|c|c|c|}
\hline $\mathrm{C}$ & 6.54789 & -1.19527 & -0.42297 \\
\hline $\mathrm{C}$ & 6.08619 & 0.10677 & -0.49382 \\
\hline $\mathrm{S}$ & 7.43519 & 1.23008 & -0.5278 \\
\hline $\mathrm{C}$ & 2.18843 & 0.75503 & -0.59274 \\
\hline $\mathrm{C}$ & 2.8456 & 1.97399 & -0.6356 \\
\hline $\mathrm{C}$ & 4.26404 & 1.8792 & -0.6038 \\
\hline $\mathrm{C}$ & 4.7238 & 0.57635 & -0.53525 \\
\hline $\mathrm{S}$ & 3.36878 & -0.53681 & -0.50795 \\
\hline $\mathrm{C}$ & 21.77918 & -1.03491 & -0.16387 \\
\hline $\mathrm{H}$ & -17.815 & -2.52211 & 0.33374 \\
\hline $\mathrm{H}$ & -20.4044 & -2.644 & -0.06585 \\
\hline $\mathrm{H}$ & -16.389 & 1.66936 & -1.73833 \\
\hline $\mathrm{H}$ & -13.812 & 1.80422 & -1.35187 \\
\hline $\mathrm{H}$ & -10.0664 & -2.13174 & 1.4817 \\
\hline $\mathrm{H}$ & -12.6419 & -2.26541 & 1.09295 \\
\hline $\mathrm{H}$ & -8.6513 & 2.08202 & -0.55477 \\
\hline $\mathrm{H}$ & -6.07426 & 2.21451 & -0.16654 \\
\hline $\mathrm{H}$ & -2.31649 & -1.73737 & 2.61965 \\
\hline $\mathrm{H}$ & -4.89277 & -1.88427 & 2.22074 \\
\hline $\mathrm{H}$ & -22.0919 & 0.43344 & -1.05454 \\
\hline $\mathrm{H}$ & -22.3418 & -1.31963 & -1.16543 \\
\hline $\mathrm{H}$ & -21.8131 & -0.40703 & -2.59202 \\
\hline $\mathrm{H}$ & 0.33762 & -1.49486 & 2.58491 \\
\hline $\mathrm{H}$ & -0.19691 & -0.39287 & 3.85339 \\
\hline $\mathrm{H}$ & -0.89486 & 3.1222 & 1.46528 \\
\hline $\mathrm{H}$ & 0.71517 & 2.7978 & 2.09999 \\
\hline $\mathrm{H}$ & -0.77892 & 3.80765 & 3.88162 \\
\hline $\mathrm{H}$ & -0.28558 & 2.17009 & 4.34743 \\
\hline $\mathrm{H}$ & -1.91448 & 2.45579 & 3.70365 \\
\hline $\mathrm{H}$ & 2.30034 & -0.7074 & 3.95012 \\
\hline $\mathrm{H}$ & 1.92186 & 0.97939 & 3.56271 \\
\hline $\mathrm{H}$ & 2.46033 & -0.12314 & 2.28443 \\
\hline $\mathrm{H}$ & -0.17401 & 2.8434 & -1.17437 \\
\hline $\mathrm{H}$ & -1.54881 & 1.79566 & -1.50145 \\
\hline $\mathrm{H}$ & -0.5248 & 2.64528 & -3.65579 \\
\hline $\mathrm{H}$ & -0.38769 & 0.88158 & -3.5609 \\
\hline $\mathrm{H}$ & 1.02594 & 1.89776 & -3.2216 \\
\hline $\mathrm{H}$ & 0.35716 & -1.26992 & -2.32554 \\
\hline $\mathrm{H}$ & -1.20018 & -1.19613 & -1.49982 \\
\hline $\mathrm{H}$ & -0.04774 & -3.37347 & -0.99563 \\
\hline $\mathrm{H}$ & -0.23426 & -2.41664 & 0.48595 \\
\hline $\mathrm{H}$ & 1.34338 & -2.51251 & -0.31982 \\
\hline $\mathrm{H}$ & 17.95902 & 1.79399 & -0.43787 \\
\hline $\mathrm{H}$ & 20.57362 & 1.59228 & -0.37863 \\
\hline $\mathrm{H}$ & 16.30713 & -2.79661 & -0.13811 \\
\hline $\mathrm{H}$ & 13.70479 & -2.61276 & -0.19293 \\
\hline $\mathrm{H}$ & 10.13945 & 2.35444 & -0.60307 \\
\hline $\mathrm{H}$ & 12.74012 & 2.16755 & -0.54624 \\
\hline $\mathrm{H}$ & 8.48895 & -2.24035 & -0.35007 \\
\hline $\mathrm{H}$ & 5.88673 & -2.05039 & -0.3916 \\
\hline $\mathrm{H}$ & 2.3201 & 2.91774 & -0.69375 \\
\hline $\mathrm{H}$ & 4.92314 & 2.73655 & -0.63651 \\
\hline $\mathrm{H}$ & 22.00898 & -1.7152 & -0.993 \\
\hline $\mathrm{H}$ & 21.98406 & -1.56458 & 0.77465 \\
\hline $\mathrm{H}$ & 22.45846 & -0.17929 & -0.22728 \\
\hline
\end{tabular}

$5 \mathrm{~T}_{3}$ (Sum of electronic and thermal Enthalpies $=-9784.977936$ Hartree $)$

\begin{tabular}{|l|c|c|c|}
\hline & $\mathrm{X}$ & $\mathrm{Y}$ & $\mathrm{Z}$ \\
\hline $\mathrm{C}$ & 1.42532 & 21.97919 & -0.20953 \\
\hline $\mathrm{C}$ & 0.04899 & 12.20384 & -1.69912 \\
\hline $\mathrm{C}$ & -0.17769 & 10.80645 & -1.68047 \\
\hline $\mathrm{C}$ & -0.41887 & 10.29878 & -0.41537 \\
\hline $\mathrm{S}$ & -0.36448 & 11.58664 & 0.77795 \\
\hline $\mathrm{C}$ & 0.16352 & 14.17921 & -0.0999 \\
\hline $\mathrm{C}$ & 0.11031 & 14.77035 & 1.15005 \\
\hline $\mathrm{C}$ & 0.33528 & 16.16929 & 1.12762 \\
\hline $\mathrm{C}$ & 0.56422 & 16.67501 & -0.13929 \\
\hline $\mathrm{S}$ & 0.5006 & 15.38709 & -1.33032 \\
\hline $\mathrm{C}$ & -0.01472 & 12.79428 & -0.44853 \\
\hline $\mathrm{C}$ & -1.20932 & 4.47605 & -1.61306 \\
\hline $\mathrm{C}$ & -1.44735 & 3.07337 & -1.5963 \\
\hline $\mathrm{C}$ & -1.69065 & 2.54954 & -0.33775 \\
\hline
\end{tabular}




\begin{tabular}{|c|c|c|c|}
\hline $\mathrm{S}$ & -1.62706 & 3.83571 & 0.84993 \\
\hline $\mathrm{C}$ & -1.08777 & 6.44235 & -0.00264 \\
\hline $\mathrm{C}$ & -1.14141 & 7.02749 & 1.24974 \\
\hline $\mathrm{C}$ & -0.91539 & 8.42598 & 1.23488 \\
\hline $\mathrm{C}$ & -0.68428 & 8.93773 & -0.0304 \\
\hline $\mathrm{S}$ & -0.75003 & 7.65423 & -1.22712 \\
\hline $\mathrm{C}$ & -1.2673 & 5.05604 & -0.3576 \\
\hline $\mathrm{Si}$ & -1.96144 & 0.74952 & 0.13482 \\
\hline $\mathrm{C}$ & 1.23174 & 20.53028 & -0.56725 \\
\hline $\mathrm{C}$ & 1.2772 & 19.95254 & -1.81111 \\
\hline $\mathrm{C}$ & 1.04911 & 18.54461 & -1.79576 \\
\hline $\mathrm{C}$ & 0.8268 & 18.03817 & -0.53121 \\
\hline $\mathrm{S}$ & 0.90217 & 19.33034 & 0.65931 \\
\hline $\mathrm{C}$ & -3.16229 & -0.00079 & -1.12151 \\
\hline $\mathrm{C}$ & -2.70275 & 0.69929 & 1.87721 \\
\hline $\mathrm{C}$ & -20.405 & -9.02872 & -0.69695 \\
\hline $\mathrm{C}$ & -10.7064 & -6.7505 & -1.39261 \\
\hline $\mathrm{C}$ & -9.3588 & -6.33162 & -1.27758 \\
\hline $\mathrm{C}$ & -9.07418 & -5.69586 & -0.08121 \\
\hline $\mathrm{S}$ & -10.5122 & -5.61851 & 0.92431 \\
\hline $\mathrm{C}$ & -12.8762 & -6.71412 & -0.06365 \\
\hline $\mathrm{C}$ & -13.6524 & -6.39801 & 1.03743 \\
\hline $\mathrm{C}$ & -15.0012 & -6.81586 & 0.92145 \\
\hline $\mathrm{C}$ & -15.2832 & -7.45914 & -0.27016 \\
\hline $\mathrm{S}$ & -13.8429 & -7.54967 & -1.26967 \\
\hline $\mathrm{C}$ & -11.4805 & -6.44354 & -0.28651 \\
\hline $\mathrm{C}$ & -3.2896 & -4.26298 & -0.81808 \\
\hline $\mathrm{C}$ & -1.94305 & -3.81786 & -0.69938 \\
\hline $\mathrm{C}$ & -1.63306 & -3.25388 & 0.52701 \\
\hline $\mathrm{S}$ & -3.04884 & -3.28944 & 1.55724 \\
\hline $\mathrm{C}$ & -5.42804 & -4.35933 & 0.56082 \\
\hline $\mathrm{C}$ & -6.1901 & -4.09915 & 1.68562 \\
\hline $\mathrm{C}$ & -7.53223 & -4.53868 & 1.57833 \\
\hline $\mathrm{C}$ & -7.82349 & -5.14348 & 0.36776 \\
\hline $\mathrm{S}$ & -6.39734 & -5.17189 & -0.65704 \\
\hline $\mathrm{C}$ & -4.04066 & -4.04488 & 0.32415 \\
\hline $\mathrm{Si}$ & -0.01761 & -2.4509 & 1.05757 \\
\hline $\mathrm{C}$ & -18.9438 & -8.74875 & -0.92334 \\
\hline $\mathrm{C}$ & -18.1802 & -9.07208 & -2.01672 \\
\hline $\mathrm{C}$ & -16.8202 & -8.65679 & -1.90676 \\
\hline $\mathrm{C}$ & -16.5379 & -8.0101 & -0.72052 \\
\hline $\mathrm{S}$ & -17.984 & -7.91549 & 0.2756 \\
\hline $\mathrm{C}$ & 1.39924 & -3.56425 & 0.47981 \\
\hline $\mathrm{C}$ & -0.01748 & -2.30458 & 2.94646 \\
\hline $\mathrm{C}$ & 19.03715 & -11.6045 & -1.42921 \\
\hline $\mathrm{C}$ & 11.17865 & -6.12565 & 1.39434 \\
\hline $\mathrm{C}$ & 10.04389 & -5.29634 & 1.56541 \\
\hline $\mathrm{C}$ & 9.55831 & -4.76714 & 0.38198 \\
\hline $\mathrm{S}$ & 10.53083 & -5.31469 & -0.97428 \\
\hline $\mathrm{C}$ & 12.68739 & -7.00238 & -0.4579 \\
\hline $\mathrm{C}$ & 13.09531 & -7.12086 & -1.77485 \\
\hline $\mathrm{C}$ & 14.23233 & -7.94875 & -1.94589 \\
\hline $\mathrm{C}$ & 14.71593 & -8.47969 & -0.76372 \\
\hline $\mathrm{S}$ & 13.73763 & -7.94238 & 0.59117 \\
\hline $\mathrm{C}$ & 11.5842 & -6.24834 & 0.07628 \\
\hline $\mathrm{C}$ & 4.93122 & -1.47816 & 2.33761 \\
\hline $\mathrm{C}$ & 3.7957 & -0.63401 & 2.49844 \\
\hline $\mathrm{C}$ & 3.29155 & -0.12323 & 1.3144 \\
\hline $\mathrm{S}$ & 4.25097 & -0.71141 & -0.02905 \\
\hline $\mathrm{C}$ & 6.41486 & -2.40085 & 0.48631 \\
\hline $\mathrm{C}$ & 6.80735 & -2.54305 & -0.83249 \\
\hline $\mathrm{C}$ & 7.93804 & -3.37882 & -1.00308 \\
\hline $\mathrm{C}$ & 8.43281 & -3.8926 & 0.18328 \\
\hline $\mathrm{S}$ & 7.46965 & -3.3289 & 1.53935 \\
\hline $\mathrm{C}$ & 5.31707 & -1.63146 & 1.018 \\
\hline $\mathrm{Si}$ & 1.77632 & 0.95729 & 1.0543 \\
\hline $\mathrm{C}$ & 17.87658 & -10.822 & -0.87602 \\
\hline $\mathrm{C}$ & 17.47376 & -10.718 & 0.43162 \\
\hline $\mathrm{C}$ & 16.32698 & -9.88941 & 0.612 \\
\hline $\mathrm{C}$ & 15.84629 & -9.35354 & -0.56562 \\
\hline $\mathrm{S}$ & 16.83345 & -9.88649 & -1.91994 \\
\hline $\mathrm{C}$ & 2.24183 & 2.40871 & -0.06838 \\
\hline $\mathrm{C}$ & 1.24614 & 1.58352 & 2.76177 \\
\hline $\mathrm{Si}$ & 0.11165 & -0.34301 & 0.03624 \\
\hline
\end{tabular}




\begin{tabular}{|c|c|c|c|}
\hline $\bar{C}$ & 0.57345 & -0.57898 & -1.81143 \\
\hline $\mathrm{H}$ & 2.27556 & 22.11624 & 0.46983 \\
\hline $\mathrm{H}$ & 0.53447 & 22.39694 & 0.27532 \\
\hline $\mathrm{H}$ & 1.62001 & 22.55577 & -1.11906 \\
\hline $\mathrm{H}$ & 0.25492 & 12.76276 & -2.60187 \\
\hline $\mathrm{H}$ & -0.16293 & 10.18811 & -2.56779 \\
\hline $\mathrm{H}$ & -0.08489 & 14.21218 & 2.05576 \\
\hline $\mathrm{H}$ & 0.32911 & 16.78894 & 2.01403 \\
\hline $\mathrm{H}$ & -1.00645 & 5.04005 & -2.51396 \\
\hline $\mathrm{H}$ & -1.43968 & 2.46383 & -2.4904 \\
\hline $\mathrm{H}$ & -1.33884 & 6.46423 & 2.15164 \\
\hline $\mathrm{H}$ & -0.9209 & 9.04071 & 2.1248 \\
\hline $\mathrm{H}$ & 1.46825 & 20.51787 & -2.71319 \\
\hline $\mathrm{H}$ & 1.049 & 17.9288 & -2.68525 \\
\hline $\mathrm{H}$ & -3.3107 & -1.07043 & -0.92851 \\
\hline $\mathrm{H}$ & -2.78126 & 0.10556 & -2.14567 \\
\hline $\mathrm{H}$ & -4.13739 & 0.50104 & -1.07047 \\
\hline $\mathrm{H}$ & -2.92614 & -0.3371 & 2.16147 \\
\hline $\mathrm{H}$ & -3.6431 & 1.26558 & 1.91598 \\
\hline $\mathrm{H}$ & -2.02062 & 1.1174 & 2.62887 \\
\hline $\mathrm{H}$ & -20.9765 & -8.10231 & -0.56114 \\
\hline $\mathrm{H}$ & -20.5627 & -9.65589 & 0.18907 \\
\hline $\mathrm{H}$ & -20.8131 & -9.55636 & -1.56453 \\
\hline $\mathrm{H}$ & -11.1003 & -7.26135 & -2.26075 \\
\hline $\mathrm{H}$ & -8.61676 & -6.49133 & -2.04815 \\
\hline $\mathrm{H}$ & -13.2611 & -5.87913 & 1.90214 \\
\hline $\mathrm{H}$ & -15.7454 & -6.6499 & 1.68849 \\
\hline $\mathrm{H}$ & -3.6933 & -4.72498 & -1.70944 \\
\hline $\mathrm{H}$ & -1.21949 & -3.90925 & -1.49876 \\
\hline $\mathrm{H}$ & -5.79291 & -3.60148 & 2.55984 \\
\hline $\mathrm{H}$ & -8.26531 & -4.41446 & 2.36385 \\
\hline $\mathrm{H}$ & -18.5795 & -9.59271 & -2.87658 \\
\hline $\mathrm{H}$ & -16.0778 & -8.82937 & -2.67458 \\
\hline $\mathrm{H}$ & 2.37015 & -3.10607 & 0.70524 \\
\hline $\mathrm{H}$ & 1.35358 & -3.73485 & -0.60362 \\
\hline $\mathrm{H}$ & 1.35044 & -4.54162 & 0.97759 \\
\hline $\mathrm{H}$ & 0.93889 & -1.8859 & 3.28631 \\
\hline $\mathrm{H}$ & -0.13874 & -3.29009 & 3.41562 \\
\hline $\mathrm{H}$ & -0.82029 & -1.6507 & 3.31156 \\
\hline $\mathrm{H}$ & 18.70561 & -12.3625 & -2.14933 \\
\hline $\mathrm{H}$ & 19.55418 & -12.1151 & -0.61102 \\
\hline $\mathrm{H}$ & 19.75932 & -10.9514 & -1.93434 \\
\hline $\mathrm{H}$ & 11.68605 & -6.61869 & 2.21243 \\
\hline $\mathrm{H}$ & 9.59457 & -5.09152 & 2.52772 \\
\hline $\mathrm{H}$ & 12.59079 & -6.62422 & -2.59266 \\
\hline $\mathrm{H}$ & 14.68512 & -8.14961 & -2.90731 \\
\hline $\mathrm{H}$ & 5.44945 & -1.95033 & 3.16189 \\
\hline $\mathrm{H}$ & 3.36634 & -0.40126 & 3.46355 \\
\hline $\mathrm{H}$ & 6.29542 & -2.05611 & -1.65133 \\
\hline $\mathrm{H}$ & 8.37868 & -3.59723 & -1.9664 \\
\hline $\mathrm{H}$ & 17.9831 & -11.2202 & 1.24295 \\
\hline $\mathrm{H}$ & 15.87359 & -9.69651 & 1.57514 \\
\hline $\mathrm{H}$ & 1.36198 & 3.02765 & -0.28361 \\
\hline $\mathrm{H}$ & 2.6488 & 2.05809 & -1.02602 \\
\hline $\mathrm{H}$ & 3.00272 & 3.03967 & 0.40974 \\
\hline $\mathrm{H}$ & 2.07141 & 2.11832 & 3.25091 \\
\hline $\mathrm{H}$ & 0.93087 & 0.76282 & 3.41833 \\
\hline $\mathrm{H}$ & 0.40646 & 2.2837 & 2.66444 \\
\hline $\mathrm{H}$ & 1.54917 & -1.07194 & -1.9087 \\
\hline $\mathrm{H}$ & 0.62847 & 0.39121 & -2.32163 \\
\hline $\mathrm{H}$ & -0.1768 & -1.19446 & -2.32387 \\
\hline
\end{tabular}

\section{References}

1. Kim D.-H.; Ohshita, J.; Kunugi, Y.; Kunai, A. Chem. Lett. 2006, 35, 266.

2. Ohshita, J.; Uemura, T.; Kim, D.-H.; Kunai, A.; Kunugi, Y.; Kakimoto, M. Macromolecules 2005, 38, 730 .

3. Kim, D.-H.; Ohshita, J.; Lee, K-H.; Kunugi, Y.; Kunai, A. Organometallics 2006, 25, 1511. 